\title{
LISTA COMENTADA E ILUSTRADA DOS CAECIDAE (MOLLUSCA, PROSOBRANCHIA, MESOGASTROPODA) DA OPERAÇÃO OCEANOGRÁFICA GEOMAR XII
}

\author{
Renata dos Santos Gomes ${ }^{1}$ \\ Ricardo Silva Absalão 1, 2
}

\begin{abstract}
Commented and illustrated list of the Caecidae (Mollusca, Prosobranchis, Mesogastropoda) From OceAnographic Operation Geomar XII. The family Caecidae deserved special attention, among mollusks, because they occurred in more than $50 \%$ of studied stations in Oceanographic Operation GEOMAR XII, which was carried out in northern and southern coasts of Rio de Janeiro and Espírito Santo States (Brazil), respectively. At the time, Caecum brasilicum Folin, 1874, C. aff. condylum Moore, 1969, C. circumvolutum Folin, 1867, C. johnsoni Winkley, 1908, C. someri Folin, 1867, C. striatum Folin, 1868, C. venosum Folin, 1867, C. floridanum Stimpson, 1851, C. massambabensis Absalão, 1994, C.butoti Jong \& Coomans, 1989, C. ryssotitum Folin, 1867, C. nitidum (Stimpson., 1851) e C. cubitatum (Folin, 1868) were identified. C. venosum is reported, for the first time, since its original description, enlarging its occurrence area from Pernambuco to Rio de Janeiro; $C$. butoti as well, primarily restricted to Dutch Antilles, begins to make part of the Brazilian malacofauna. C. someri and C. butoti were pointed out for being largely non-sintopic with other congeneric species, suggesting that subtile differences in sediment can be responsable for this segregation.

KEY WORDS. Mesogastropoda, Caecidae, Caecum, taxonomy, geographic distribution, sintopy
\end{abstract}

A Comissão Oceanográfica GEOMAR XII foi realizada para dar continuidade ao programa plurianual de Geologia e Geofísica da Marinha, estudando a morfologia e sedimentação da margem continental sudeste, ao largo dos estados do Espírito Santo e do Rio de Janeiro (Brasil). A análise biológica desse material mostrou uma alta diversidade de micromoluscos, sendo que os Caecidae se destacavam, dentre as demais famílias, pela abundância, ocorrendo em $57,14 \%$ de todas as estações, sendo por isso, motivo de interesse para esse estudo.

Os Caecidae são prosobrânquios marinhos encontrados nos interstícios da areia, em bancos de algas, de gramíneas, próximos a recifes de corais, em sedimentos calcáreos ou areno-lamosos (MELLO \& MAESTRATI 1986). Geralmente encontramse no meso-litoral ou em profundidades de 10 a 50 metros e estão amplamente distribuídos nas regiões tropicais das Américas. Os animais medem de um a cinco milímetros e apresentam partes moles com tentáculos cefálicos longos, podendo ter

1) Departamento de Zoologia, Instituto de Biologia, Universidade Federal do Rio de Janeiro. 21941-570 Rio de Janeiro, Rio de Janeiro, Brasil.

2) Pesquisador do CNPq.

Revta bras. Zool. 13 (2): 513 - 531, 1996 
olhos sésseis ou serem cegos, pé truncado e curto. A concha é pequena, tubular, curvada e aberta na extremidade anterior. A extremidade posterior é lacrada pelo septo e esculturada pelo mucro, através do qual pode-se fazer a separação da família em subgêneros (Rıos 1994): Caecum, Brochina, Elephantulum, Fartulum e Meioceras. Apresentam desenvolvimento planctotrófico, começando a vida pelo caracol típico, véliger natante com concha espiral (ABBOTT 1974). Num primeiro estágio de crescimento, a concha se encontra espiralada, mas após a perda das voltas nucleares que ocorre quando o animal passa a viver no bentos, a concha se torna cilíndrica, alcançando um segundo estágio de desenvolvimento. A seguir, forma-se um novo septo na parte posterior da concha e o segundo estágio é descartado, totalizando cerca de três estágios de desenvolvimento.

A taxonomia da família é pouco estudada e os Caecidae já chegaram a ser incluídos entre os Cephalopoda do Paleozóico e também entre os Scaphopoda, até que MOORE (1962) reconheceu-os como pertencentes à superfamília Rissoidea, baseado em similaridades encontradas nas partes moles, estabelecendo uma separação dos Vermetidae-Cerithidae, conforme se aceita desde 1855. O autor que mais publicou no Atlântico Oeste foi Folin, descrevendo 64 espécies. Das 19 espécies assinaladas para a costa brasileira, segundo RIos (1994), 10 foram descritas por Folin.

Não só os Caecidae do Brasil precisam de uma revisão taxonômica, mas também, todo o material procedente do Atlântico Oeste (ABSALÃo 1995; ABSALÃo \& GOMES 1995). Este trabalho, por restringir-se aos Caecidae coligidos durante a Operação Oceanográfica GEOMAR XII, pretende ser mais um passo em direção à revisão dessa família no Brasil.

\section{MATERIAL E MÉTODOS}

A Comissão Oceanográfica GEOMAR XII foi realizada pelo Navio Oceanográfico Almirante Câmara, da Diretoria de Hidrografia e Navegação, no período compreendido entre agosto e setembro de 1979, para estudar a morfologia e sedimentação da margem continental do sudeste, ao largo dos Estados do Espírito Santo e Rio de Janeiro. Para isso foram coligidas amostras geológicas, das quais obtiveram-se 112 amostras que não haviam sofrido o processo usual de descalcificação que precede as análises sedimentológicas. O material malacológico foi triado sob magnificação e conservado a seco. Inicialmente os Caecidae foram separados em morfo-espécies e fotografados em microscopia eletrônica de varredura e, em seguida, determinados taxonomicamente segundo FoLIN (1867a,b, 1887), ABBOTT (1974), Rios (1985, 1994), LeAl (1990) e LightFoot (1992 a,b). Utilizou-se, sempre que possível, a comparação direta com material depositado no Instituto de Biologia da Universidade Federal do Rio de Janeiro, Museu Oceanográfico Eliézer de Carvalho Rios e com o material cedido por empréstimo do Museu de Zoologia de Amsterdam e do Museu de História Natural de Paris. Todo o material desse estudo está depositado na Coleção de Moluscos do Departamento de Zoologia, Instituto de Biologia, Universidade Federal do Rio de Janeiro (UFRJ). 


\title{
Abreviações utilizadas no texto
}

Muséum National d'Histoire Naturelle (MNHN), Paris; Museu Oceanográfico Prof. Eliézer de Carvalho Rios (MORG), Rio Grande; The Natural History Museum (BMNH), Londres; University of Miami, Marine Laboratory (UMML); Zoologisch Museum Amsterdam (ZMA); Instituto de Biologia da Universidade Federal do Rio de Janeiro (IB-UFRJ), Rio de Janeiro; Navio Oceanográfico Almirante Câmara (NOAC); Navio Oceanográfico Almirante Saldanha (NOAS).

\section{RESULTADOS}

\section{Taxonomia}

\author{
Caecidae Gray, 1850 \\ Caecum (Caecum) Fleming, 1813 \\ Caecum brasilicum Folin, 1874
}

Figs 1-2

Caecum brasilicum Folin, 1874: 212. -Mello \& Maestrati, 1986: 151, fig. 6.

Caecum pulchellum Stimpson, 1851: 113. -Leal, 1990: 85, pl. 13, fig. F. - Lightfoot, 1992a: 173?, fig.

2. - Merlano \& Hegedes, 1994: 140, fig. 484.

Caecum brasilicum; Rios, 1994: 56, pl. 18, fig. 1994.

Concha pequena, creme, opaca, regularmente curvada, ornada com cerca de 24 (22-26) anéis. Em perfil os anéis da região posterior são triangulares e seguindo em direção anterior estes se tornam quadrangulares. Interespaços com a mesma largura dos anéis; exceto pelo primeiro e segundo anéis da região anterior, que são mais próximos entre si, e o terceiro e quarto anéis, que são ligeiramente mais espaçados. Anéis triangulares com interespaços lisos, anéis quadrangulares com interespaços cobertos por microestrias longitudinais. É comum as microestrias estenderem-se por sobre os anéis quadrangulares nos exemplares pouco desgastados. Excepcionalmente, pode-se encontrar indivíduos com todos os anéis quadrangulares. Septo mamilado. O mucro é uma projeção triangular e rombuda.

Material examinado. Série-tipo MNHN, Brasil; UMML, Flórida, USA, 25-VIII-1988, J.H. Leal leg.; MORG 7705, Ilhas Virgens, 1960, G. Usticke leg.; IB-UFRJ 1806, Espírito Santo I \#6446, 22-VIII-1984, NOAS leg.; MORG 25641, Ilha de Trindade, 15-X-1987, Barcellos e Laurino leg.; MORG 25629, Ilha de Trindade, 15-X-1987, Barcellos e Laurino leg.; MORG 25 293, Praia do Forte, Bahia, V-1987, Barcellos leg.; MORG 23138, Itaipú, Rio de Janeiro, 1982, R. Absalão leg.; MORG 18292, Ilha de Santo Amaro, São Paulo, V-1971, J. Vaz leg.; MORG 16776, Perequê, São Paulo, 1940, O. de Fiore leg.; MORG 18470, Porto Bello, Santa Catarina, V-1974, Eq. Oceanologia leg; MORG 18547, Itaorna, Rio de Janeiro, VII-1972, B. Helena leg.; MORG 18096, Itaorna, Rio de Janeiro, 1972; MORG 21083, Búzios, Rio de Janeiro, 9-X-1979; MORG 18053, Abrolhos, Bahia, VII-1972; MORG 21 195, Abrolhos, Bahia, I-1980, Eq. MORG leg.; MORG 12491, Recife da Marinha, Alagoas; MORG 29065, Aracruz, Espírito Santo, 1-VIII-1988, Vera Abud leg.; MORG 16773, Boqueirão, Ubatuba, São Paulo, 6-V-1958, J. de 


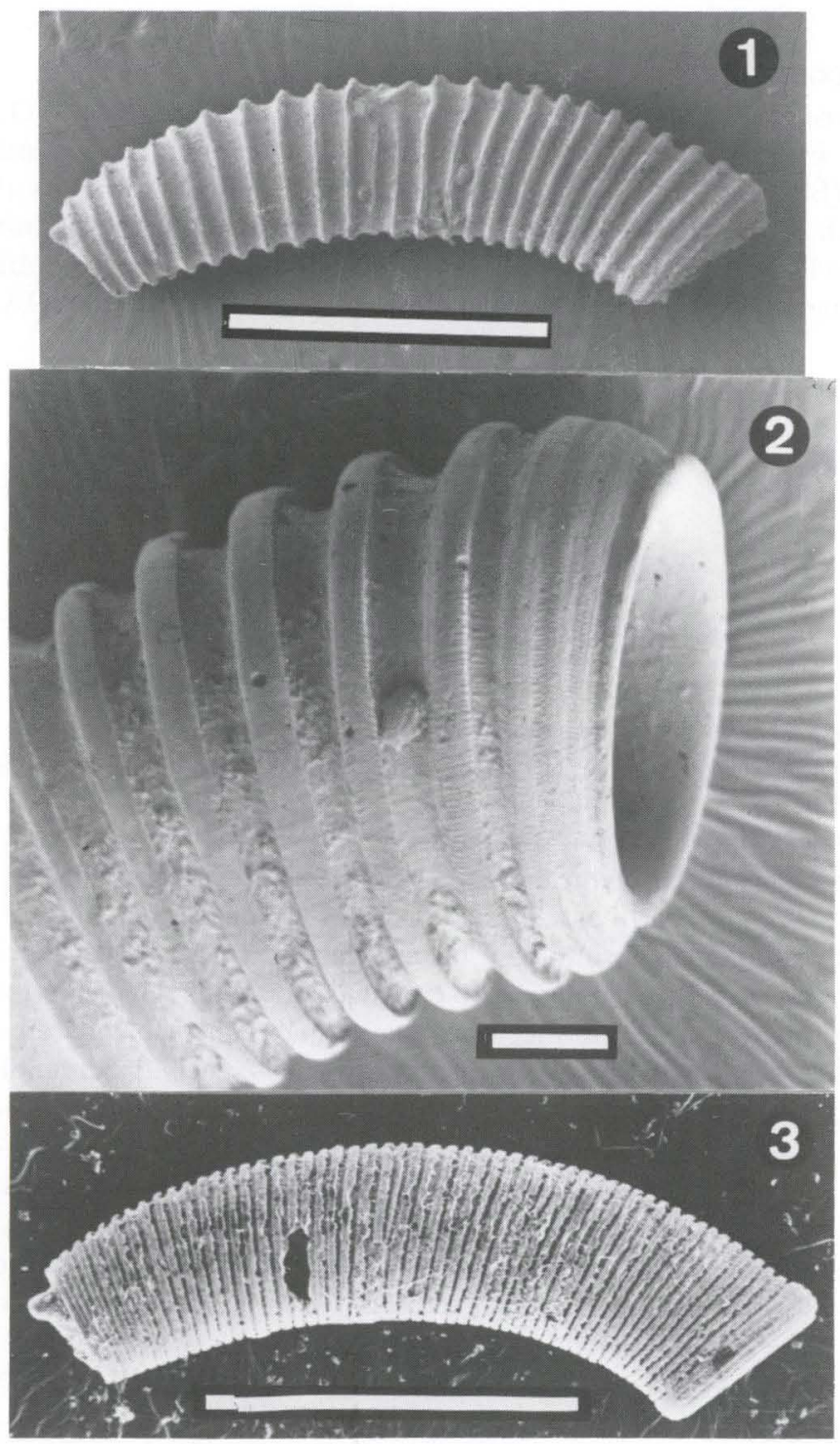

Figs 1-3. (1-2) Caecum brasilicum; (3) C. aff. condylum. Escala $=1 \mathrm{~mm}$, exceto na figura $2=$ $0,1 \mathrm{~mm}$.

Paiva Carvalho leg.; MORG 15753, Canion do Amazonas, Amazonas, 1970, NOAS leg.; MORG 24629, Fernando de Noronha, Pernambuco, 5-XII-1985, M. Cabeda leg.; MORG 27927, Prainha, Cabo Frio, Rio de Janeiro, 11-XII-1988, Tito Almeida leg.; MORG 28662, Bacia de Campos, Rio de Janeiro, 14-VII-1990, Salgueiro leg.; MORG 28757, Bacia de Campos, Rio de Janeiro, 15-VII-1990, Salgueiro leg.; 
MORG 23137, Itaipú, Rio de Janeiro, 1982, R. Absalão leg.; MORG 22825, Angra dos Reis, Rio de Janeiro, Eq. MNRJ leg..

\section{Caecum aff. condylum Moore, 1969}

Fig. 3

Caecum condylum Moore, 1969: 26. - Jong \& Coomans, 1988: 37, pl. 14, fig. 69. - Rios, 1994: 56, pl. 18, fig. 200.

Concha média, creme, opaca e moderadamente curvada, com curvatura mais acentuada na região anterior. O diâmetro da concha é praticamente o mesmo em toda ela ou aumenta muito pouco em direção anterior. Esculturada por cerca de 64 anéis que em vista lateral se apresentam quadrangulares. Estrias longitudinais microscópicas percorrem toda a extensão da concha incluindo anéis e interespaços. Presença de suave variz terminal. Septo mamilado, pouco elevado e o mucro é uma projeção dorsal e rombuda, tombada para a direita.

Material examinado. IB-UFRJ 1741, Espírito Santo I \#6422, 16-VIII-1984, NOAS leg.; Homeótipo ZMA 2445, Aruba, R. Moolembeeck leg..

\section{Caecum (Brochina) Gray, 1857 \\ Caecum circumvolutum Folin, 1867}

Fig. 4

Caecum circumvolutum Folin, 1867: 29. -Mello \& Maestrati, 1986: 155, fig. 10. - Rios, 1994: 56, pl. 18, fig. 204.

Caecum vestitum Folin, 1870: 183. - Leal, 1990: 86, pl. 13, figs J-K.

Concha de tamanho médio, cilíndrica, branca, algo translúcida. Lisa ou com fracas linhas de crescimento. Curvatura moderada, pouco mais acentuada na abertura. Região anterior com fracas ou nenhuma anelação seguida de forte variz arredondada. Septo mamilado e o mucro como uma forte projeção rombuda.

Material examinado. IB-UFRJ 6784, N-NE \#1902, 1968, NOAS leg.; MORG 26454, Sapata - Cabeda, XII-1985; MORG 20627, Fernando de Noronha, Pernambuco, I-1979, Eq. MORG leg..

\section{Caecum johnsoni Winkley, 1908}

Fig. 5

Caecum johnsoni Winkley, 1908: 54. -Keeler, 1981: 71, fig. 20). - Mello \& Maestrati, 1986: 156, fig. 11. -Lightfoot, 1992b: 28, fig. 32. - Rios, 1994: 56, pl. 18, fig. 205.

Concha média, branca, opaca e irregularmente curvada, o último terço da concha é acentuadamente mais curvado. O calibre da concha aumenta suave mas regularmente em direção anterior. Lisa em sua maior parte, mas com linhas circulares próximas da abertura. Sob microscopia eletrônica de varredura é possível observar a presença de microestrias axiais na região anterior. Região posterior suavemente comprimida lateralmente, região anterior acentuadamente comprimida, formando uma abertura oblonga com suave constricção. Septo hemisférico. Mucro é uma pequena projeção latero-dorsal rombuda.

Material examinado. MORG - referência extraviada (1 lote). 

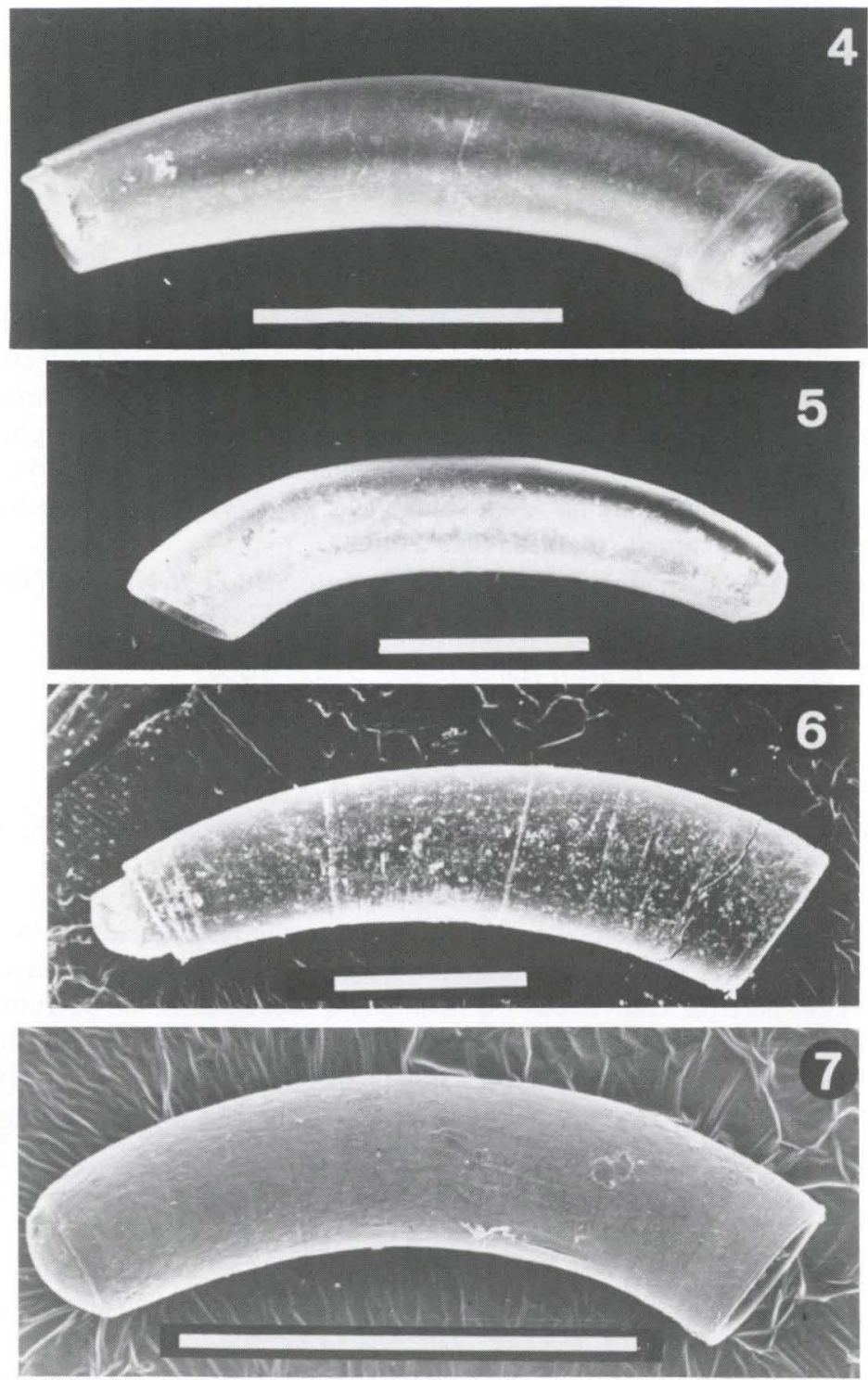

Figs 4-7. (4) Caecum circumvolutum; (5) C. johnsoni; (6) C. someri; (7) C. striatum. Escala = $1 \mathrm{~mm}$.

\section{Caecum someri Folin, 1867}

Fig. 6

Caecum someri Folin, 1867: 56. - Mello \& Maestrati, 1986: 157, fig. 12.

Caecum achironum Folin, 1867: 57. -Lightfoot, 1992b: 24, fig. 24. - Rios, 1994: 56, pl. 18, fig. 202. 
Concha média, lisa, branca e muito pouco curvada. O calibre da concha aumenta suave e regularmente da região posterior em direção à região anterior. Ausência de variz terminal ou qualquer inchaço. O septo é ungüiculado e o mucro é uma pequena projeção dorsal.

Material examinado. Série-tipo MNHN, PE-Brasil; IB-UFRJ 1670, Geocosta Rio II \#c 01, Rio de Janeiro, 24-III-1986; IB-UFRJ 948, Itaipú, Rio de Janeiro, 1981; IB-UFRJ 5934, Camburi \#5, Espírito Santo, 17-III-1992, Eq. Zoologia UFRJ leg.; IB-UFRJ 1864, Espírito Santo I \#6421, 16-VIII-1984, NOAS leg.; MORG 15814, Canion do Amazonas, Amazonas, 1970, NOAC leg.; MORG 29060, Aracruz, Espírito Santo, 1-VIII-1988, Vera Abud leg.; MORG 23136, Itaipú, Rio de Janeiro, 1982, R. Absalão leg.; MORG 21233, Abrolhos, Bahia, I-1980, Eq. MORG leg.; MORG 21814, Cabo Orange, Amapá, I-1981, Riobaldo leg.; MORG 22149, Itaipú, Rio de Janeiro, 1981, R. Absalão leg.; MORG 25232, Praia Vermelha, Rio de Janeiro, 12-I-1984, Tito leg.; MORG 21328, Marambaia, Rio de Janeiro, 1973; MORG 25292, Praia do Forte, Bahia, V-1987, L. Barcellos leg. .

\section{Caecum striatum Folin, 1868}

Fig. 7

Caecum striatum Folin, 1868: 49. - Mello \& Maestrati, 1986: 158, fig. 14. - Rios, 1994: 56, pl. 18, fig. 207.

Concha pequena, com periostraco amarronzado, translúcida e moderadamente curvada. Região anterior com diâmetro suavemente maior que o restante da concha. Concha aparentemente lisa ao microscópio ótico, exceto pelas linhas de crescimento; ao microscópio eletrônico visualizam-se microestrias longitudinais irregulares. Região posterior da concha, próximo ao septo ligeiramente estrangulada. Septo hemisférico e o mucro é uma pequena projeção latero-dorsal rombuda.

Material examinado. Série-tipo MNHN, Bahia, Pernambuco, Brasil e Panamá; IB-UFRJ 949, Itaipú, Rio de Janeiro, 1981, R. Absalão leg.; IB-UFRJ 5947, Rio de Janeiro, Rio de Janeiro, 1993, Fábio Pitombo leg.; IB-UFRJ 1865, Espírito Santo I \#6421, 16-VIII-1984, NOAS leg.; MORG 26422, Abrolhos, Bahia, II-1987, A. Silveira leg.; MORG 23139, Itaipú, Rio de Janeiro, 1982, R. Absalão leg.; MORG 26 739, Parcel do Carpinteiro, RS, 24-XI-1988, Atlântico Sul leg..

\section{Caecum venosum Folin, 1867}

Figs 8-9

Caecum venosum Folin, 1867: 46.

Caecum heladum Olsson \& Harbison, 1953: 318. - Keeler, 1981: 71, fig. 19. - Lightfoot, 1992b: 24, fig. 25.

Concha média, branca, translúcida, moderadamente curvada, cilíndrica e ligeiramente inflada na região anterior à abertura. $\mathrm{O}$ calibre é praticamente o mesmo em toda a extensão da concha. Esculturada com cordões longitudinais que percorrem toda a concha incluindo o inchaço. Em microscopia eletrônica de varredura, observam-se finas linhas longitudinais entre e sobre esses cordões principais. Presença de microesculturas anelares mais visíveis na região da abertura produzindo um padrão levemente reticulado. Septo hemisférico e o mucro é uma pequena projeção latero-dorsal rombuda. 
Material examinado. Série - tipo MNHN, Pernambuco, Brasil, 1867; MORG 24400, Ilha de Trindade, Espírito Santo, III-1986.

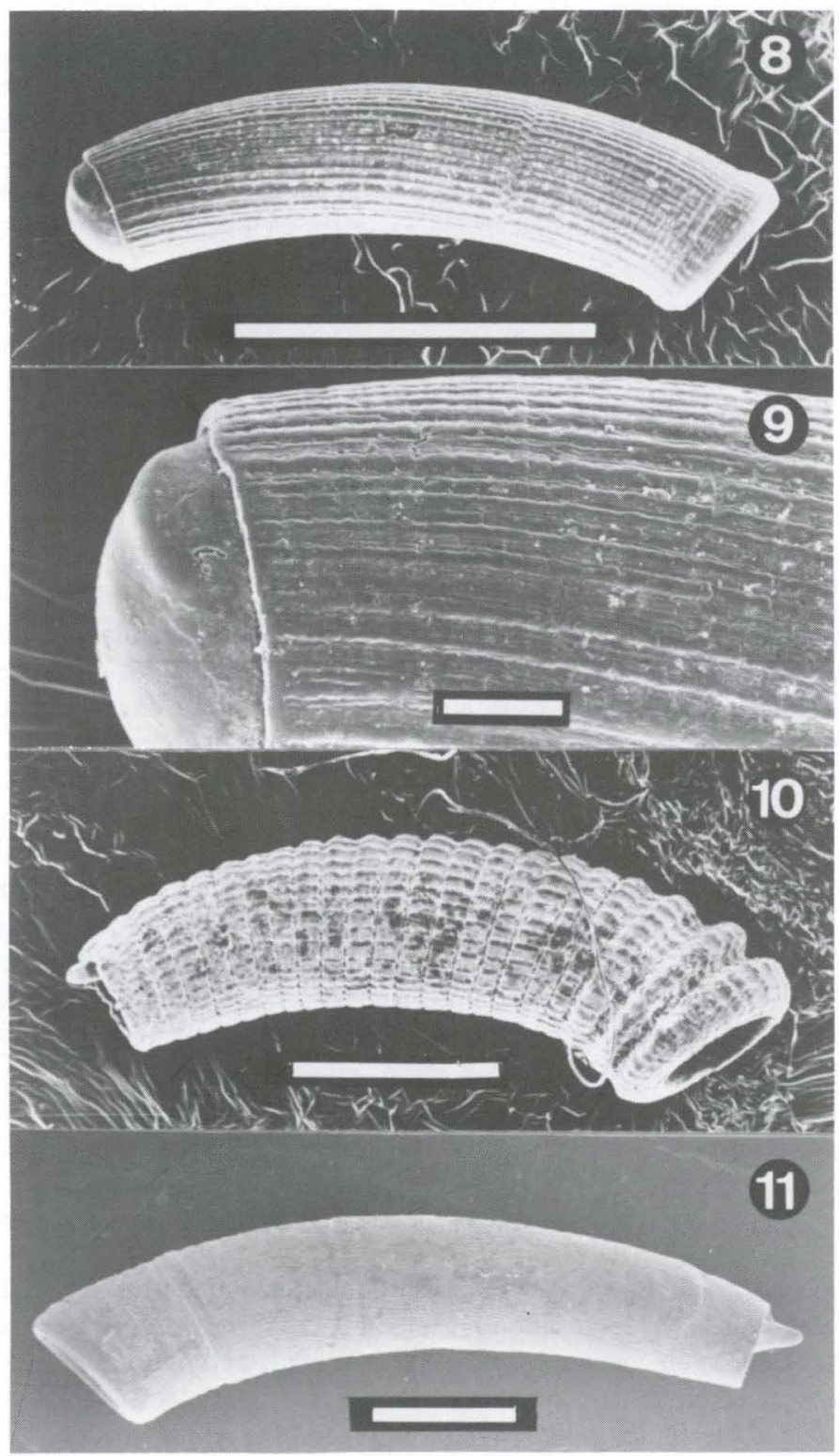

Figs 8-11. (8-9) Caecum venosum; (10) C. floridanum; (11) C. massambabensis. Escala $=$ $1 \mathrm{~mm}$, exceto na figura $9=0,1 \mathrm{~mm}$. 


\section{Caecum (Elephantulum) Carpenter, 1857 Caecum floridanum Stimpson, 1851}

Fig. 10

Caecum floridanum Stimpson, 1851: 112. -Moore, 1970: 371, fig. 2 A-B. - Keeler, 1981: 372, fig. 11. Mello \& Maestrati, 1986: 154, fig. 8. - Leal, 1990: 85, pl. 13, fig. D-E. - Rios, 1994: 57, pl. 18, fig. 211. -Merlano \& Hegedes, 1994: 141, fig. 489.

Concha grande, branca, opaca e irregularmente curvada; curvatura mais acentuada na região anterior, alguns exemplares chegam a ser quase retos. Escultura formada por anéis fortes e largos que quase se tocam. O número de anéis fica em torno de 26 (22-35). Inicialmente os interespaços são muito estreitos, quase como sulcos, mas vão aumentando de largura em direção a abertura chegando a ter a mesma largura que os últimos anéis. Presença de forte variz terminal e abertura ovalada. Apresentam finos cordões axiais que percorrem toda a extensão da concha, passando sobre anéis e interespaços. Septo retraído. O mucro é uma projeção cilíndrica e pontiaguda.

Material examinado. IB-UFRJ 6906, Aruba, 1995, F. Verberne leg.; MORG 17502, Egmont Key, Flórida; MORG 12492, Alagoas, 1964, P. Sá Cardoso leg.; MORG 16517, Foz do Amazonas, Amazonas, 8-XI-1970, NOAC leg.; MORG 20113, Abrolhos, Bahia, II-1978, Eq. MORG leg.; MORG 26418, I. Guarita, Abrolhos, Bahia, II-1987, Silveira leg.; MORG 15902, Pará, 1970, NOAC; MORG 15902, Rio Pará, 1970, NOAS leg.; MORG 18052, Abrolhos, Bahia, VII-1972, L. Araújo leg.; MORG 17502, Egmont Key, Flórida.

\section{Caecum massambabensis Absalão, 1994}

Fig. 11

Caecum massambabensis Absalão, 1994: 138.

Concha grande, opaca, sólida, moderadamente curvada. O calibre aumenta suavemente, mas de maneira constante em direção à abertura. Abertura circular com constricção muito suave. Concha toda esculturada com microestrias axiais interrompidas por linhas de crescimento irregularmente distribuídas. Septo retraído e mucro formado por uma projeção cilíndrica, estreita e pontiaguda.

Material examinado. Holótipo: IB-UFRJ 5518, ao largo da Praia de Massambaba, Rio de Janeiro, 27-III-1984, Aviso Oceanográfico Subof. Oliveira leg.; IB-UFRJ 5519, ao largo da Praia de Massambaba, Rio de Janeiro, 27-III-1984, Aviso Oceanográfico Subof. Oliveira leg.; IB-UFRJ 5520, ao largo da Praia de Massambaba, Rio de Janeiro, 27-III-1984; MORG 30588, ao largo da Praia de Massambaba, Rio de Janeiro, 27-III-1984, Aviso Oceanográfico Subof. Oliveira leg.; MZUSP 27915, ao largo da Praia de Massambaba, Rio de Janeiro, 27-III-1984, Aviso Oceanográfico Subof. Oliveira leg.; MZUSP 27916, ao largo da Praia de Massambaba, Rio de Janeiro, 27-III-1984, Aviso Oceanográfico Subof. Oliveira leg.; MNRJ 6882, ao largo da Praia de Massambaba, Rio de Janeiro, 27-III-1984, Aviso Oceanográfico Subof. Oliveira leg.; MORG 30590, ao largo da Praia de Massambaba, Rio de Janeiro, 27-III-1984, Aviso Oceanográfico Subof. Oliveira leg.; MNRJ 6883, ao largo da Praia de Massambaba, Rio de Janeiro, 27-III-1984, 
Aviso Oceanográfico Subof. Oliveira leg.; IB-UFRJ 5521, ao largo da Praia de Massambaba, Rio de Janeiro, 27-III-1984, Aviso Oceanográfico Subof. Oliveira leg.; IB-UFRJ 5522, ao largo da Praia de Massambaba, Rio de Janeiro, 27-III-1984, Aviso Oceanográfico Subof. Oliveira leg.; IB-UFRJ 5523, ao largo da Praia de Massambaba, Rio de Janeiro, 27-III-1984, Aviso Oceanográfico Subof. Oliveira leg.; IB-UFRJ 5524, ao largo da Praia de Massambaba, Rio de Janeiro, 27-III-1984, Aviso Oceanográfico Subof. Oliveira leg.; IB-UFRJ 5525, ao largo da Praia de Massambaba, Rio de Janeiro, 27-III-1984, Aviso Oceanográfico Subof. Oliveira leg..

\section{Caecum (Fartulum) Carpenter, 1857 \\ Caecum butoti Jong \& Coomans, 1988}

Fig. 12

Caecum butoti Jong \& Coomans, 1988: 39, fig. 177.

Concha média, lisa, amarronzada com manchas e bandas transversais brancas distribuídas em toda a sua extensão. Curvatura moderada, sendo acentuada na região que antecede a abertura. O calibre da concha aumenta mais acentuadamente no sexto próximo ao septo, seguindo com aumento muito suave no restante. Abertura com suave constricção. Septo mamilado e o mucro é uma projeção triangular e pontuda.

Material examinado. IB-UFRJ 5531, Espírito Santo I \#6444: 20³8’12"S 40¹6'12"W, 23-VIII-1984, R. Absalão leg.; IB-UFRJ 5530, Espírito Santo I \#6444, VIII-1984, NOAS leg.; IB-UFRJ 6499, Aruba, West Indies.

Caecum ryssotitum Folin, 1867

Fig. 13

Caecum ryssotitum Folin, 1867: 46. -Mello \& Maestrati, 1986: 159, fig. 15. - Lightfoot, 1992b: 30, fig. 36. -Rios, 1994: 57, pl. 19, fig. 214.

Caecum antillarum Carpenter, 1857. -Merlano \& Hegedes, 1994: 141, fig. 486.

Concha pequena, branca, lisa (algumas vezes com fracas ondulações ou finíssimas linhas anelares), semi-translúcida e moderadamente curvada. Calibre aumenta marcadamente na porção próxima ao septo, seguindo com aumento muito suave no restante da concha. A abertura é fortemente oblíqua e constricta. Septo mamilado. O mucro é uma projeção triangular, rombuda e dorsal.

Material examinado. Série-tipo MNHN, Mar das Antilhas, Brasil e Golfo do México; IB-UFRJ 947, Itaipú, Rio de Janeiro, R. Absalão leg.; IB-UFRJ 1874, Espírito Santo I \#6421, 16-VIII-1984, NOAS leg.; IB-UFRJ 1930, Camburí, Espírito Santo, 1986, Eq. Zoologia leg.; IB-UFRJ 3429, Itaipú, Rio de Janeiro, 1989; IB-UFRJ 1485, Moleque I, Espírito Santo, V-1985, Eq. Zoologia leg.; MORG 23137, Itaipú, Rio de Janeiro, 1982, R. Absalão leg.; MORG 21236, Abrolhos, Bahia, I-1980, Eq. MORG leg.; MORG 25261, Praia do Forte, Bahia, V-1987, L. Barcellos leg.; MORG 29057, Aracruz, Espírito Santo, 1-VIII-1988, Vera Abud leg.; MORG 21948, Itaorna, Rio de Janeiro, VII-1972, B. Helena leg.; MORG 15854 Canion do Amazonas, Amazonas, 1970, NOAS leg.; MORG 25226, Capuba 


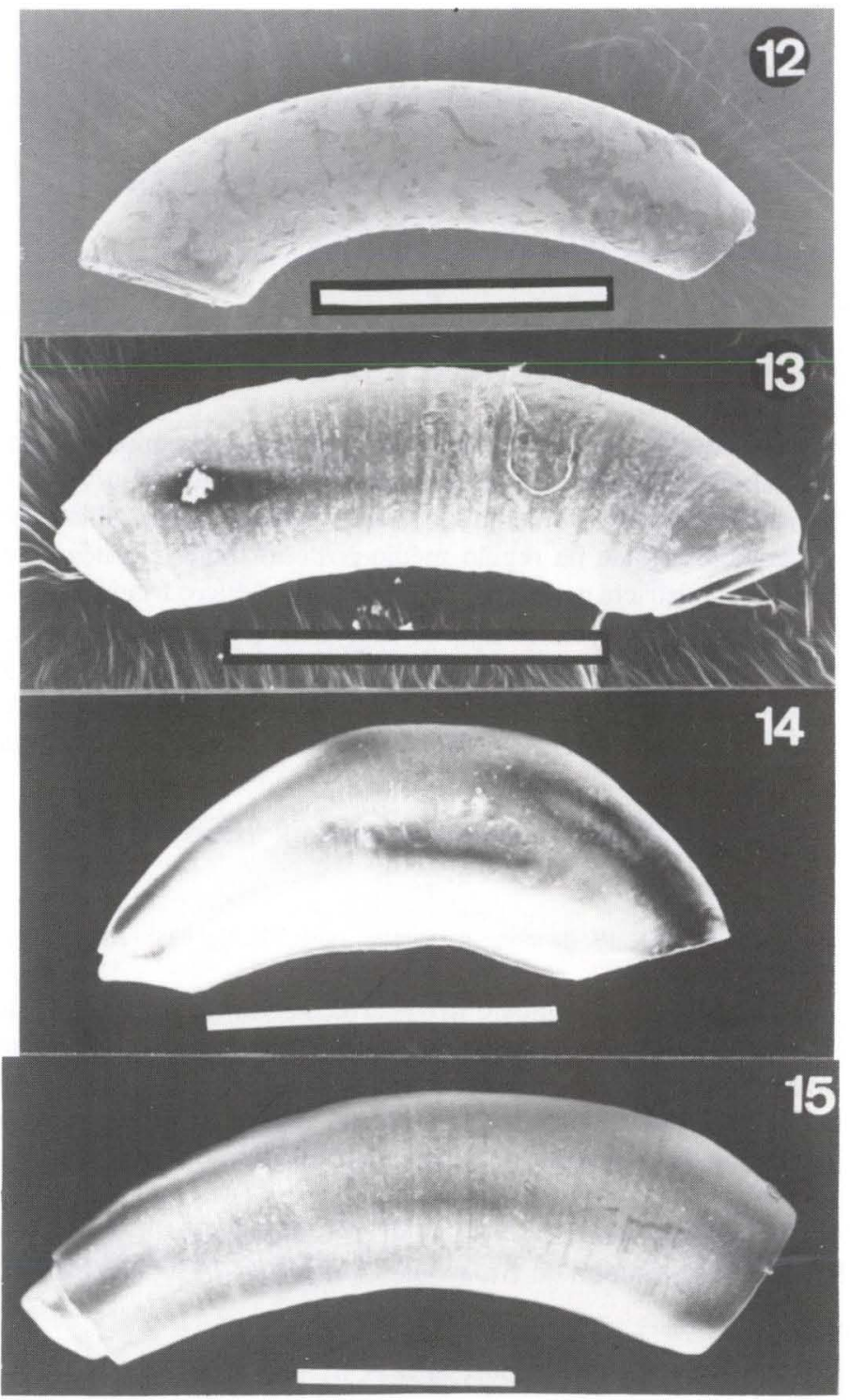

Figs 12-15. (12) Caecum butoti; (13) C. ryssotitum; (14) C. nitidum; (15) C. cubitatum. Escala= $1 \mathrm{~mm}$.

-ES, II-1986, R. Cruz leg.; MORG 5312 Porto Belo, Santa Catarina, 5-X-1952, L. de Morretes leg.; MORG 23833, Abrolhos, Bahia, I-1985, Eq. MORG leg.; MORG 21946, Ilha de Santo Amaro, São Paulo, V-1971, J. Vaz leg.; MORG 26421, Abrolhos, Bahia, II-1987, A. Silveira leg.; MORG 12993, Rec. Marinha, Alagoas, 1964, P. Sá Cardoso leg.; MORG 18591, São Vicente, São Paulo, VII-1974, J. 
Colella leg.; MORG 18842, Porto Bello, Santa Catarina, V-1974; MORG 24215, Itaipú, Rio de Janeiro, 1982, R. Absalão leg.; MORG 22825, Angra dos Reis, Rio de Janeiro, 1984, Equipe MNRJ leg..

\section{Caecum (Meioceras) Carpenter, 1858 \\ Caecum nitidum (Stimpson, 1851)}

Fig. 14

Caecum nitidum Stimpson, 1851: 112; Moore, 1972: 892, fig. 11. -Keeler, 1981: 71, fig. 22. -Mello \& Maestrati, 1986: 161, fig. 16. - Rios, 1994: 58, pl. 19, fig. 217. - Merlano \& Hegedes, 1994: 142, fig. 493.

Concha pequena, lisa, com coloração variando de amarelado ocre a amarronzado, dorsalmente há presença de manchas esbranquiçadas. Curvatura acentuada. Região posterior estreita aumentando, muito rapidamente, o calibre em direção anterior. Aparência inflada na região médio-posterior onde alcança o seu maior calibre. Abertura constricta e oblíqua. Septo elevado. Mucro triangular.

Material examinado. Série-tipo BMNH 1858 12931, West Indies; BMNH 1858 12930, Barbados; IB-UFRJ 6496, Aruba - West Indies; MORG 21235, Abrolhos, Bahia, I-1980, Equipe MORG leg.; MORG 24204, P. Areia, Abrolhos, Bahia, I-1985, Equipe MORG leg.; MORG 7704, St. Croix, Ilhas Virgens, R.D. Moore leg..

\section{Caecum cubitatum (Folin, 1868)}

Fig. 15

Caecum cubitatum Folin, 1868: 19. -Merlano \& Hegedes, 1994: 142, fig. 494. - Rios, 1994: 58, pl. 19, fig. 216.

Concha média, lisa, branca e irregularmente curvada, com curvatura mais acentuada na região anterior. O calibre da concha aumenta de maneira acentuada em direção anterior atingindo o seu máximo a cerca de $1 / 4$ da abertura. A partir dessa altura ocorre uma suave constricção antes de chegar a abertura. Presença de esporádicas linhas de crescimento. Septo ungüiculado e o mucro é uma pequena projeção dorsal.

Material examinado. IB-UFRJ 4150, Amasseds \#3210, Amazonas, 12-V1990; MORG 15814, Canion do Amazonas, Amazonas, 1970, NOAC leg..

\section{Distribuição}

Dos 13 táxons determinados, apenas $C$. someri (com ocorrência em 29 estações), C. brasilicum (com ocorrência em 24 estações), C. butoti (com ocorrência em 16 estações), C. nitidum (com ocorrência em 15 estações) e C. ryssotitum (com ocorrência em 13 estações), tiveram uma freqüência mínima de modo a permitir uma análise.

A figura 16 mostra a distribuição destas cinco espécies na área abrangida pela GEOMAR XII. Nota-se que há apenas uma ocorrência abaixo de Lagoa Feia. Aparentemente, uma mudança no tipo sedimentológico poderia estar associada a esta ausência, pois o sedimento predominante nas estações abaixo da referida região é siltoso (Md 4,44 Ø), enquanto que o acima da Lagoa Feia é arenoso (Md 1,32 Ø). 


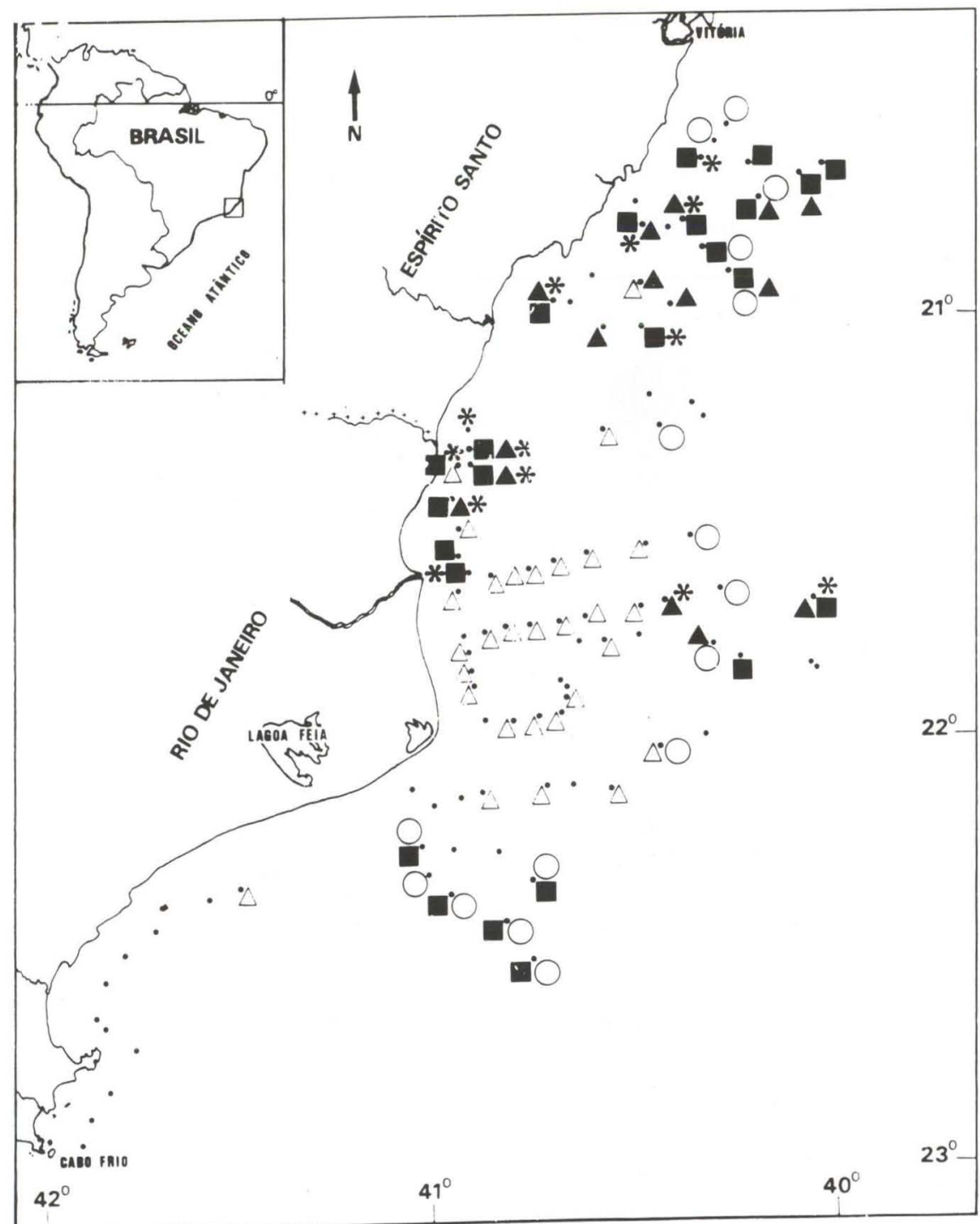

Fig. 16. Vista parcial da área abrangida pela Comissão Oceanográfica GEOMAR XII, onde as cinco espécies mais freqüentes de Caecum estiveram presentes: triângulo vasado: $C$. someri; triângulo cheio: C. nitidum; quadrado cheio: C. brasilicum; círculo vasado: $C$. butotie asterisco: C. ryssotitum.

Pode-se reunir as cinco espécies em três grupos de acordo com o grau de exclusividade de sua área de ocorrência (Fig. 17); destaca-se C. someri com cerca de $90 \%$ de sua área de ocorrência exclusiva, não sendo compartilhada (sintopia) com nenhuma outra espécie da família. Segue-se C. butoti com quase metade de sua 
área total de ocorrência sendo exclusiva, e finalmente C. brasilicum, C. nitidum e C. ryssotitum com, respectivamente, 20,83, 20,00 e 7,70\% de suas áreas sendo exclusivas.

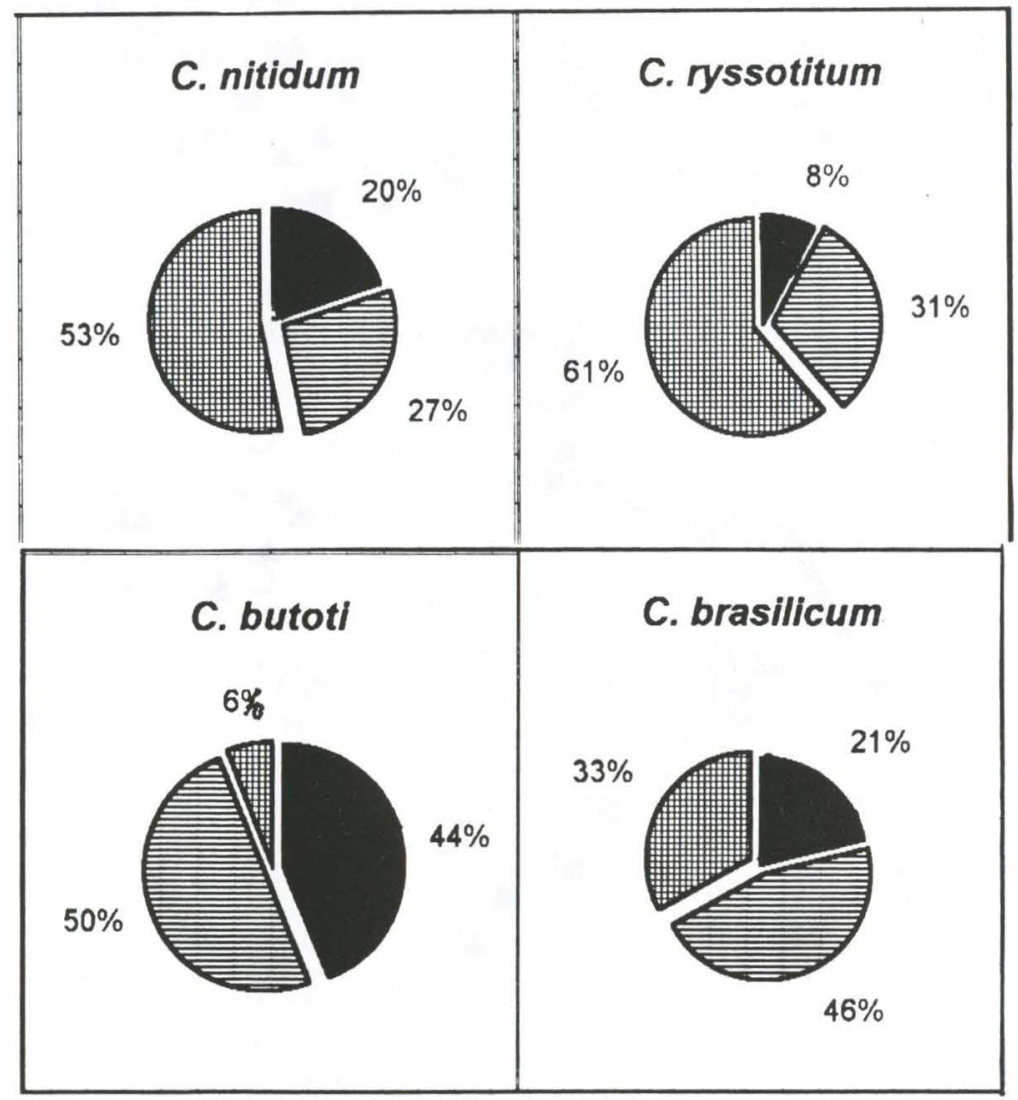

Fig. 17. Os gráficos representam o padrão de ocorrência das cinco espécies principais: o padrão escuro representa a percentagem de ocorrência em alopatria; o listrado, a percentagem de ocorrência com apenas uma espécie congenérica; e o padrão quadriculado, a percentagem de ocorrência com duas ou mais espécies congenéricas.

\section{DISCUSSÃO}

\section{Aspectos Taxonômicos}

A espécie mais próxima de $C$. brasilicum é $C$. pulchellum já que ambas apresentam o mesmo número de anéis, tipo de septo e ausência de inchaço ou variz à abertura; no entanto, a descrição de $C$. pulchellum não faz referência a presença de microestrias nos interespaços, que se faziam presentes em nossos exemplares. Essa característica foi assinalada por Folin em sua descrição original de C. brasili- 
cum. O exame da série-tipo de C. brasilicum revelou que a maioria dos indivíduos apresentam dois tipos de anéis em vista lateral: os triangulares e os quadrangulares (Fig. 1). Quando ambos os tipos de anéis estão presentes num mesmo indivíduo, os anéis quadrangulares distribuem-se na metade anterior da concha. As microestrias restringem-se aos anéis quadrangulares (Fig. 2), sendo mais visíveis nos interespaços, enquanto os anéis triangulares se apresentam lisos. Assim, um exame restrito da região posterior das conchas de C. brasilicum e C. pulchellum não permite a separação entre elas e somente sob forte magnificação pode-se distingüir as estrias longitudinais da primeira. Por outro lado, C. pulchellum sempre apresenta todos os anéis triangulares em vista lateral e ausência completa de ornamentação longitudinal.

A determinação de C. aff. condylum por Rios (1994, p.56) foi apenas tentativa como ele mesmo reconhece. A descrição e ilustração de MOORE (1969) são suficientemente claras para saber-se que estamos tratando com espécies distintas, o que o exame do homeótipo confirmou. $\mathrm{O}$ verdadeiro C. condylum tem cerca de 100 anéis; enquanto que nos exemplares examinados por nós, o número de anéis variou de 51 à 90 , com um valor médio igual a 64, incluindo-se dois ou três anéis situados sobre a variz terminal. C. condylum apresenta um inchaço próximo à abertura, o que não foi observado em nenhum dos nossos exemplares de $C$. aff. condylum. Por outro lado, C. aff. condylum mostrou a existência de uma variz terminal ausente na outra espécie. Em suma, C. aff. condylum é, sem dúvida, uma espécie distinta de $C$. condylum e, provavelmente, ainda não descrita formalmente.

Dentre as espécies do subgênero Brochina, C. striatum se assemelha muito à C. strigosum. C. striatum não apresenta qualquer tipo de inchaço ou constricção próximo à abertura, sendo o seu calibre praticamente o mesmo em toda a sua extensão. Ambas as espécies são esculturadas por microestrias longitudinais visíveis à microscopia eletrônica. C. striatum apresenta ainda, um estrangulamento na região próxima ao septo.

Caecum strigosum, por outro lado, apresenta um pequeno, mas claro inchaço precedendo à abertura. Este inchaço foi muito acentuado por Folin ao ilustrar a espécie, de modo que ao se tratar com exemplares jovens se torna muito difícil diferenciar as duas espécies, especialmente porque a constricção próxima ao septo que $C$. striatum apresenta, pode estar ausente em alguns espécimens.

Após sua descrição em 1867, baseada em concha quebrada, C. venosum com localidade-tipo assinalada para o Estado de Pernambuco, Brasil, não foi mais reportado para o litoral brasileiro até que ABSALÃo (1995) fotografasse o tipo e determinasse o homeótipo. C. venosum (Fig. 8) embora apresente o mesmo tamanho, calibre, septo, mucro e curvatura que C. striatum (Fig. 7) e C. strigosum, tem como traço distintivo a forte esculturação longitudinal composta por cordões e finas linhas, enquanto que as espécies precedentes mostram apenas estrias longitudinais microscópicas. É possível que o que Rios (1994, p.57) nomeia de C. heladum Olsson \& Harbison, 1953 se trate, na verdade, de C. venosum e, neste caso, a sinonímia de C. heladum (sensu Rios) sob C. striatum seria inteiramente equivocada. Caecum (Brochina) jonhsoni (Fig. 5) apresenta concha mais estreita e longa em relação às três espécies citadas acima. O aumento de calibre em direção anterior é 
mais visível e a curvatura é mais acentuada na porção anterior próxima à abertura, onde ocorrem fracas anelações. MELlo \& MAestrati (1986) e Rios (1994) descrevem o septo de C. jonhsoni como possuidor de estrias em arco de círculo. Esta característica está ausente em nossos espécimens e também não pode ser observada no único exemplar depositado no Museu Oceanográfico do Rio Grande; além disso, Mello (comunicação pessoal) confirmou a inexistência desta ornamentação nos exemplares examinados por ela.

Caecum someri (Fig. 6) tem sido sinonimizado por Rios (1985 e 1994) sob C. achironum; por outro lado, MELLO \& MAESTRATI (1986) reconhecem-nas como espécies distintas. A diferença básica entre elas consiste em finíssimas estrias longitudinais presentes em C. achironum e ausentes em C. someri, como Folin (1867) ao descrevê-las apontava. Assim, mantêm-se o status de espécie válida para C. someri.

O exame do holótipo de C. butoti (ZMA 3.87.067) mostrou que o mesmo apresenta semelhança superficial com C. ryssotitum, uma espécie reconhecidamente polimórfica como demonstrado por sua abundante sinonímia. No entanto, os topótipos e homeótipos depositados na mesma instituição sugerem que o holótipo não retrata bem a forma média típica da espécie. Além disso, C. ryssotitum (Fig. 13) é geralmente desprovido de cor, enquanto C. butoti (Fig. 12) apresenta-se amarronzado com manchas brancas bem evidentes. C. butoti é sensivelmente menos inflado e constricto à abertura que $C$. ryssotitum. C. butoti foi recentemente assinalado para a costa brasileira por ABSALÃO \& GOMES (1995).

Caecum cubitatum (Fig. 15), como aqui ilustrado, está de acordo com RIOS (1994, pl. 19; fig. 216) e é bastante distinto das ilustrações de KEELER (1981, p.72; fig. 24) e LightFOOT (1992b, p.30; fig. 35). Aparentemente C. cubitatum sensu RIOS (1994) apresenta uma possível gradação à C. someri; no entanto estas questões serão aprofundadas num trabalho próximo que revise os Caecidae do Brasil.

\section{Aspectos da Distribuição}

O sedimento siltoso, aparentemente, representou uma barreira eficiente à ocorrência dos Caecidae nas estações mais ao sul, a despeito de MELLO \& MAESTRATI (1986) apontarem esse tipo sedimentológico como um dos habitats normalmente utilizados pela família. A batimetria dessas estações mais ao sul é, essencialmente, a mesma de várias outras onde os Caecidae estiveram presentes ao norte da Lagoa Feia descartando-se, assim, a possibilidade de uma barreira batimétrica aos Caecidae como um todo.

A distribuição basicamente não sintópica de $C$. someri leva a considerações sobre os motivos desta não co-ocorrência com as espécies congenéricas. Poderia-se especular se não se trataria de um caso de exclusão competitiva onde $C$. someri por ser um competidor superior eliminaria seus co-genéricos em $90 \%$ de sua área de ocorrência. Esta hipótese enfrenta dois fortes pontos de oposição: 1. falta de disponibilidade de dados bio-ecológicos que corroborem ou refutem tal hipótese. Na verdade a ecologia dos Caecidae é muito pouco conhecida, sendo Moore (1972) uma das poucas exceções; além disso WIENS (1989) ressalta que para caracterizar a ocorrência de competição interespecifica deve-se demonstrar que as espécies 
sobrepõem-se na utilização dos recursos e que a competição sobre estes recursos tem efeitos negativos; 2. todas as outras quatro espécies principais (na verdade também sete das oito espécies restantes) co-ocorreram com congenéricas, o que evidencia ainda mais a "exceção à regra" em que C. someri se constituiria. Por outro lado, SCHOENER $(1974,1986)$ compilou dados de muitos estudos que mostram a ocorrência de segregação ecológica; mas mesmo que tais segregações tenham ocorrido através de modificações evolutivas no passado, permanece em aberto a questão da competição interespecífica estar atuando hoje em dia.

Uma alternativa à hipótese anterior seria postular que o padrão de não sintopia apresentado principalmente por $C$. someri e secundariamente por $C$. butoti seja devido a diferenças encontradas no habitat dessas espécies que os tornassem menos apropriados à maioria das demais. KREBS (1994) apresenta vários exemplos compilados que mostram que na maioria absoluta dos casos que se julgava constituirem-se sobreposições de nichos entre espécies filogeneticamente próximas havia diferenças, nem sempre tão sutis, nos habitat de cada espécie. Assim, ao examinarse a sedimentologia das estações onde as cinco espécies principais ocorreram constata-se que embora, na média, o sedimento onde todas se encontravam fosse classificado como areia média, havia ligeira distinção entre o ambiente sedimentar onde C. someri e C. butoti habitavam (Fig. 16). Caracterizando-se por ser ligeiramente mais grosseiro para C. butoti e ligeiramente mais fino para C. someri, justamente as duas espécies com maiores percentagens de ocorrências não sintópicas. Explicando-se, desta maneira, a menor sintopia dessas espécies não como decorrência de exclusão competitiva, mas por uma repartição mais fina do ambiente sedimentar. Por sua vez, este tem grande influência sobre os organismos bentônicos (THORSON 1957; RHOADS \& YOUNG 1970; ABSALÃo 1990, 1991), mas especialmente sobre os Caecidae que são, majoritariamente, intersticiais. Quanto a capacidade de perceber "diferenças sutis" do habitat sedimentar WEBB (1969) mostrou que em areias marinhas submersas classificadas, pela metodologia usual, como areias "puras" podem conter até $10 \%$ de outros tipos granulométricos e que percentuais de 1 a $5 \%$ de lama alteravam, de maneira significativa, a permeabilidade do sedimento de modo a ser detectada por anfioxos.

Quanto à maioria das espécies que foram amplamente sintópicas, não raro, com mais de uma espécie, permanece a questão em aberto até que dados sobre a microdistribuição e/ou dietas alimentares estejam disponíveis.

AgRadecimentos. Ao Dr. Serge Gofas do Museu de História Natural de Paris, pelo empréstimo dos tipos descritos por Folin; ao Dr. Robert Moolembeeck do Museu de Zoologia de Amsterdam, pelo empréstimo dos tipos de Caecum butoti; ao Dr. Jose Henrique Leal da Universidade de Miami, pelo empréstimo do tipo de Caecum condylum e do exemplar de Caecum pulchellum. Ao Prof. Eliézer de Carvalho Rios do Museu Oceanográfico Prof. Eliézer de Carvalho Rios, Rio Grande, pelo envio de parte da literatura e de material malacológico suplementar. Ao biólogo Paulo Márcio Costa, pela confecção do mapa; ao biólogo Paulino Soares de Souza Júnior, pelas sugestões ao texto final; ao Dr. Eduardo Koutsoukos, diretor do SEBIPE-DIVEX, CENPES -PETROBRȦS, pelo acesso ao microscópio eletrônico de varredu- 
ra; ao LAGEMAR -UFF pelo acesso aos dados sedimentológicos. A Fundação Universitária

José Bonifácio (UFRJ) e ao CNPq que suportaram parcialmente este trabalho.

\section{REFERÊNCIAS BIBLIOGRÁFICAS}

Aввот, R.T. 1974. American Seashells. $2^{\text {nd }}$ ed. Van Nostrand Reinhold, 663p.

ABSALÃO, R.S. 1990. Ophiuroid Assemblages of the Lagoa dos Patos Outlet, Southern Brazil. Ophelia 31 (2): 133-143.

1991. Environmental Discrimination among Soft - Bottom Mollusc Association off Lagoa dos Patos, South, Brazil. Estuarine, Coastal and Shelf Science 32: 71-86.

Mesogastropoda) from southern Brazil. J. Conch. Lond. 35: 137-140.

1995. Sobre semelhanças conquiliológicas entre Caecum strigosum,

Folin, 1867 e Caecum striatum Folin, 1868. Resumos II Congresso Latino Americano de Malacologia, p.50.

AbSALÃO, R.S. \& R.S. Gomes. 1995. Ocorrência de Caecum butoti Jong \& Coomans no Brasil. Biociências 3 (1): 207-211.

Folin, L. DE. 1867a. Le genre Meioceras. Ann. Soc. Linn. Maine et Loire 11: $17-31$.

15: $44-58$.

1867b. Descriptions d'especes nouvelles de Caecidae. J. Conch. Lond. 1887. Les Fonds de La Mer. Paris, 1258p..

JONG, K.M. \& H.E. COOMANS. 1989. Marine Gastropods from Curaçao, Aruba and Bonaire. E.J. Brill Publish, 261p.

KeELER, J.H. 1981. Minute Shells: Western Atlantic Caecids. Festivus 13 (6): $67-72$.

KreBS, C.J. 1994. Ecology. New York, Harper Collin Pub..

LEAL, J.H. 1990. Marine Prosobranch Gastropods from Oceanic Island off

Brazil: Species composition and Biogeography. Ph.D. Thesis, not publicated, University of Miami, Miami, 418p.

Lightfoot, J. 1992a. Caecidae of the Western Atlantic. Of Sea and Shore 14 (4): 171- 185.

1992b. Caecidae of the Western Atlantic. Part 2, Conclusion. Of Sea and Shore 15 (1): 23-32.

Mello, R.L.S. \& P. Maestrati. 1986. A Família Caecidae Gray, 1850 no Nordeste do Brasil. Cadeira Omega da Universidade Federal Rural de Pernambuco, Sér. Ciências Aquáticas (2): 145-166.

Merlano, J.M.D. \& M.P. Hegedes. 1994. Moluscos del Caribe Colombiano. Santa Fé de Bogotá, Fund. Natura, 365p.

Moore, D.R. 1962. The Systematic Position of the Family Caecidae (Mollusca:

Gastropoda). Bull. Mar. Sci. Gulf Caribbean 12 (4): 695-701.

83 (1): 26-28. 
1970. A new Caecum from Puerto Rico and the Virgin Island. Bull. Mar. Sci. 20 (2): 368-373.

1972. Ecological and Systematic Notes on Caecidae from St. Croix, U.S. Virgin Island. Bull. Mar. Sci. 22 (4): 881-899.

Olsson, A.A.; A. Harbison. 1953. Pliocene Mollusca of Southern Florida. Acad. Nat. Scien. Phil. 8: 1-457.

RHOADS, D.C. \& D.K. Young. 1970. The influence of the deposit feeding organisms on sediment stability and community trophic structure. Jour. Mar. Res. 28: 150-178.

Rios, E.C. 1985. Seashells of Brazil. Rio Grande, Museu Oceanográfico Eliézer de Carvalho Rios, Fundação Universidade do Rio Grande, 328p.

. 1994. Seashells of Brazil. Rio Grande, Museu Oceanográfico Eliézer de Carvalho Rios, Fundação Universidade do Rio Grande, 368p.

Shoener, T.W. 1974. Competition and the form of habitat shift. Theor. Popul. Biol. 6: 265-307.

- 1986. Resource Partitioning, p.91-126.In: J. KIKKAWA \& D. J. ANDERSON (Ed.). Community Ecology: Pattern and Process. Melbourne, Blackwell.

Thorson, G. 1957. Bottom Communities (sublittoral or shallow shelf). In: J.W. Hedgreth. (Ed). Treatise on marine ecology and paleontology. Geol. Soc. Ann. Mem. 1 (67): 461-534.

WeBB, J.E. 1969. Biologically Significant Properties of Submerged Marine Sands.

Proc. R. Soc. B. 174: 355-402.

WiEns, J.A. 1989. The Ecology of Bird Communities. Processes and Variations. Cambridge, Cambridge University Press, 63p.

Recebido em 08.XII.1995; aceito em 17.X.1996. 